\title{
Evaluation of effect of a vitamin-based barrier cream on the clinical severity of actinic cheilitis: A preliminary study
}

\author{
Mariana-Sudati Rodrigues ${ }^{1}$, Eduardo-Oliveira Kaefer ${ }^{2}$, Juliana-Tomaz Sganzerla ${ }^{1}$, Humberto-Thomazi Gassen ${ }^{1}$, \\ Rubem-Beraldo dos Santos ${ }^{1}$, Sergio-Augusto-Quevedo Miguens-Jr ${ }^{1}$
}

${ }^{1}$ Lutheran University of Brazil - ULBRA (Graduate Program in Dentistry), Canoas, RS, Brazil

${ }^{2}$ Lutheran University of Brazil - ULBRA (School of Dentistry), Cachoeira do Sul, RS, Brazil

Correspondence:

Department of Oral Medicine, Graduate Program in Dentistry

Lutheran University of Brazil

Av. Farroupilha 8001, Prédio 59

Bairro São José, Canoas - RS - CEP 92425-900

samiguens@gmail.com

Received: 07/03/2020 Accepted: 02/07/2020
Rodrigues MS, Kaefer EO, Sganzerla JT, Gassen HT, dos Santos RB, Miguens-Jr SAQ. Evaluation of effect of a vitamin-based barrier cream on the clinical severity of actinic cheilitis: A preliminary study. J Clin Exp Dent. 2020;12(10):e944-50.

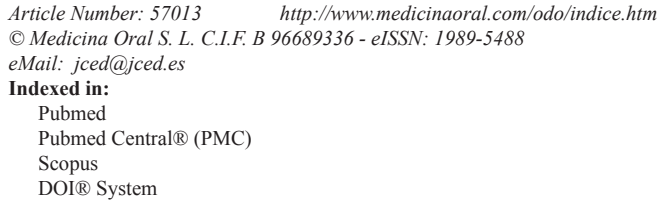

\begin{abstract}
Background: Actinic Cheilitis (AC) is a pathological condition of the labial mucosa considered potentially malignant. The aim of this study was to investigate the effect of treatment of AC with daily use of a vitamin-based barrier cream.

Material and Methods: For this clinical study, 36 participants with lower-lip AC were recruited from three oral medicine services. At baseline, participants were evaluated by clinical examination and clinical severity of AC was classified as grade I to IV. All participants were dispensed a tube of a barrier cream containing vitamins A, D, E and $\mathrm{ZnO}$ to apply once nightly for 90 consecutive days. Monthly follow-up was performed to reclassify AC clinial severity. The primary outcome of interest was clinical remission of AC at 90-day follow-up compared to baseline. Results: Progressive remission of AC lesions was observed as early as the first month and throughout the assessment period $(p=0.000)$. The 3-month period was insufficient for remission of lesions, especially among male participants $(p=0.002)$ and with a longer sun exposure in years $(p=0.007)$.

Conclusions: Daily use of the vitamin-based barrier cream had a promissing positive impact on the severity of actinic cheilitis. However, a 90-day course of treatment was not sufficient to achieve lesions remission. The findings of this study suggest a promising new avenue for the treatment of lower-lip AC.
\end{abstract}

Key words: Actinic cheilitis, vitamins, retinoids, vitamin D, therapeutics. 


\section{Introduction}

Actinic cheilitis (AC) is a pathological condition of the labial mucosa, often affecting the lower lip, which is characterized by a chronic inflammatory process caused by labial senility resulting from excessive, continuous ultraviolet radiation (UVR) exposure (1). AC is considered potentially malignant due to the risk of transformation into squamous cell carcinoma (SCC) of the lip $(2,3)$. However, there are no follow-up studies of untreated AC that would allow estimation of annual malignant transformation rates (4). The overall prevalence of QA has been reported to range between $0.45 \%$ and $2.4 \%$ (2). It is most frequent in fair-skinned males, between the fourth and fifth decades of life, with a history of occupational sun exposure $(2,5,6)$; the prevalence rises to approximately $44 \%$ in this population $(2,6)$.

The clinical appearance of AC lesions varies widely, from slight dryness or chapped lips to frank leukoplakia and/or ulcerations. The aspect of these lesions is not always consistent with the histopathological spectrum of epithelial alterations, which include atrophy, hyperkeratosis, and even dysplasia of varying degrees, which portends transformation to $\operatorname{SCC}(2,3)$. Another factor to be considered is the delay in diagnosis due to the characteristic asymptomatic nature of $\mathrm{AC}$, which has impacts on both indications for and efficacy of treatment. In addition, AC patients rarely used lip photoprotection as a prevention method (7).

Among several available treatment modalities, the most commonly used options are vermilionectomy ("lip shave"), $\mathrm{CO} 2$ laser ablation, photodynamic therapy, and chemotherapeutic agents (8-11). However, patient reports of discomfort and cosmetic and functional sequelae, especially after surgical treatment, have recently prompted the use of minimally invasive therapies. Topical medications are being investigated; these interventions are associated with a lower rate of adverse events (11).

Vitamin-based therapies have been used to reverse cutaneous photodamage and in the treatment of field cancerization $(12,13)$, with particularly promising results for vitamin A analogs. Applied topically, retinoic acid and other metabolites of vitamin A have been used to fight the signs of photoaging and are capable of reversing the photodamage caused by excessive sun exposure, especially in actinic keratoses; furthermore, they are safe for long-term use $(12,14)$. Topical use of vitamin D (cholecalciferol) and related active ingredients has been shown to reduce cell death and curtail excess proteolytic activity in the epidermis, with consequent improvement in skin hydration and, when combined with other agents, reductions in the number of actinic keratoses and their adverse effects (15-18). Vitamin E (tocopherol), an antioxidant capable of mitigating tissue damage caused by free radicals, appears promising to reduce photoinduced skin damage when used topically (19).
The used of barrier cream containing vitamins and minerals, indicated for the treatment of dermatitis, contains as its active ingredient vitamin $\mathrm{D}$ and zinc oxide ( $\mathrm{ZnO})(19$, 20). However, there have been no reports on the use of these vitamins on the lip epithelium or in the treatment of AC. Thus, the present study was designed to investigate the clinical effect of treatment of actinic cheilitis (AC) lesions with daily use of a barrier cream containing vitamins $\mathrm{A}, \mathrm{D}, \mathrm{E}$ and $\mathrm{ZnO}$.

\section{Material and Methods}

-Study design and ethical considerations

This clinical preliminary single-arm study was approved by the Research Ethics Committee of the Lutheran University of Brazil (protocol $n^{\circ} 2,751,893$ ) and conducted in accordance with National Health Council Resolution $466 / 12$.

-Study sample and setting

The sample consisted of individuals recruited from three oral medicine services located in different municipalities of south of Brazil.

Sample size calculation, with a statistical power of $80 \%$ and a significance level of $5 \%$, was estimated by a mean (SD) difference of $0.63(0.9)$ grades of AC severity from baseline after treatment, based on a pilot study by the authors. The minimum sample size was established as 30 participants. To account for possible losses to follow-up, an additional six participants were included $(\mathrm{n}=36)$.

Participants entered the study consecutively, and underwent monthly follow-up until each had individually completed a 90-day treatment period.

-Eligibility criteria

Adult participants (age $\geq 18$ years) of both sexes with a clinical diagnosis of lower-lip AC were deemed eligible. Participants with presence of leukoplakia and/or labial ulcerations, a history or treatment of head and neck cancer, previous history of skin-cancer, diabetes mellitus, immunosuppression, or known allergy to any of the components of the study drug were excluded.

-Intervention and control groups

After an interview to collect sociodemographic (gender, age, education level, household income), behavioral (sun exposure, smoking, alcohol intake), and clinical (medical and dental history) variables, participants underwent clinical examination and photographs were obtained with a 720p HDR digital camera (Apple Inc.) at 240fps for evaluation. Using a scheme adapted from Poitevin et al. (21), AC severity was classified as grade I (dryness and/or peeling of the surface of the vermilion of the lips); grade II (atrophy of the vermilion border of the lip with a slightly pale, raised surface and loss of sharpness of the boundary between skin and vermilion border, or presence of a melanotic line); grade III (any of the above, plus presence of rough and scaly areas of hyperkeratosis invading the inner mucosa of the lip); or 
grade IV (any of the above plus erosion). All procedures were performed by two previously trained examiners. The classification of lesions before the treatment intervention was intended to serve as an individual baseline control for each participant.

For treatment of $\mathrm{AC}$ lesions, each participant received a $45 \mathrm{~g}$ tube of a barrier cream containing vitamins A, D, E and $\mathrm{ZnO}$ (Hipoglós ${ }^{\circledR}$, Johnson \& Johnson) and were given verbal and written instructions to apply a thin layer to the upper and lower lips once nightly for 90 consecutive evenings. All were instructed not to eat or drink for at least 1 hour after application. Furthermore, each participant received a stick of Protective Lip Balm (SPF 30) and was instructed to apply it during the day or before any sun exposure.

Throughout the observation period, participants were asked to bring the ointment tube to each study visit for confirmation of treatment adherence. Once treatment had begun, participants returned for follow-up visits at 30,60 , and 90 days. At each visit, two examiners performed all procedures as at baseline. A third examiner an expert in oral medicine, and blinded to clinical information, reviewed all photographs to confirm the classification of AC severity grade in each participant at each time point of assessment.

Photographs were obtained at a standard distance, and resolution and viewed in a dark room on a laptop computer (Apple Inc.). Images from each time point were selected randomly by the third examiner for evaluation every 14 days. Kappa coefficients were calculated to assess intra-examiner agreement. A kappa $\geq 0.81$ (exce- llent) was considered indicative of a valid result to allow reproducibility of the $\mathrm{AC}$ lesion classification method used in the study.

Throughout the observation period, participants whose lesions progressed in severity were immediately excluded from the sample and referred for biopsy.

For analysis of the primary outcome (lesion remission) and estimation of treatment effect, the baseline grade of severity was compared with that observed at the end of follow-up (90 days). The outcome was classified as: complete remission; partial remission; or lesion unchanged (no grade change).

-Data analysis

Data were analyzed by descriptive statistics and generalized estimation equations (GEE) for longitudinal data analysis (observation period), adjusted by Bonferroni multiple comparisons. Analysis of correlation between variables was carried out by Student's t-test for up to two groups and Spearman's test, one-way ANOVA, and post-hoc tests for more than two groups. All analyses were performed in IBM SPSS Version 25.0 software. The significance level was set at $p<0.05$.

\section{Results}

Of the 36 participants recruited, 35 met the eligibility criteria and were included in the study. During the observation period, six participants were lost to follow-up: four dropped out, one died, and one participant was excluded at the 60-day visit due to lesion requiring biopsy. Thus, 29 participants completed the study (Fig. 1).

Most participants were male (54.3\%), fair-skinned

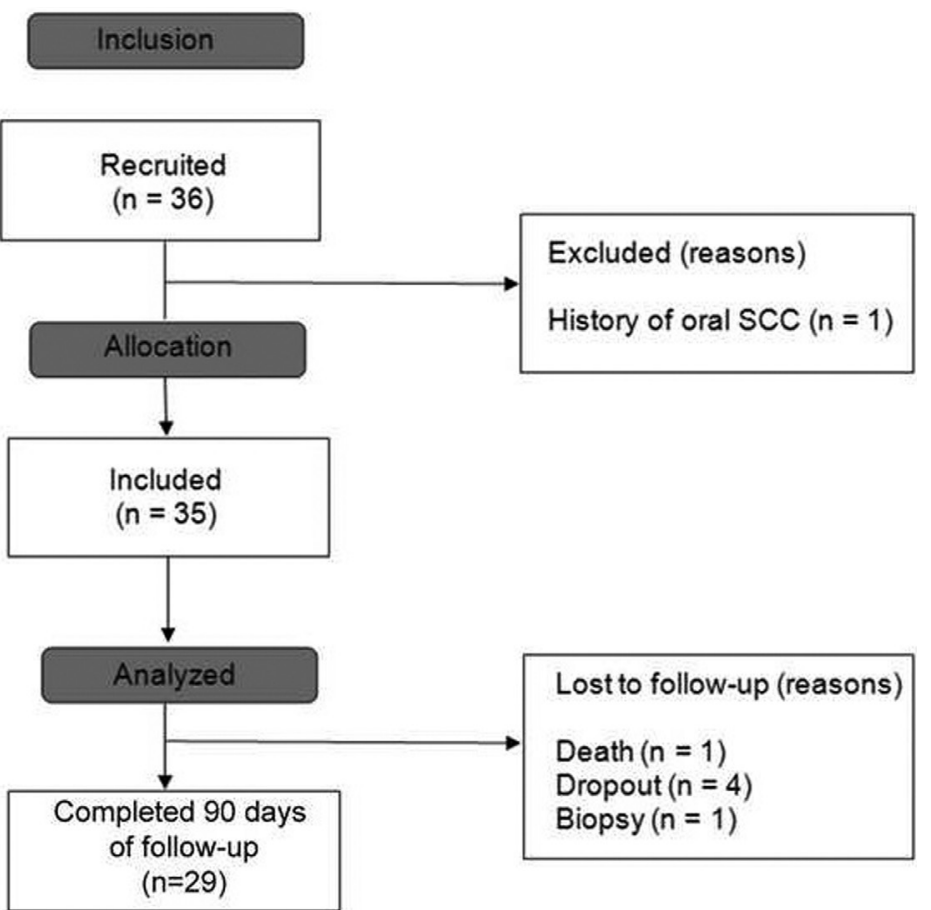

Fig. 1: Flowchart of participants selection and follow-up. 
$(91.4 \%)$, and had worked or otherwise been exposed to work in the sun $(65.7 \%)$, for at least 8 hours daily $(69.56 \%)$, over 20 years or longer $(52.2 \%)$, and had never worn sunscreen $(60 \%)$. The mean age was $56.66( \pm$ 13.66) years. Many participants had low educational attainment (high school or less; 48.6\%), a household income of 1 to 3 times the current minimum wage (37.1\%), and most reported some type of comorbidity (e.g., hypertension) (67.5\%). Regarding behavioral variables, smoking or former smoking was reported by $91.4 \%$ of the sample, while current or past alcohol intake was reported by $20 \%$ of participants (Table 1 ).
Intra-examiner agreement on the clinical severity of AC lesions was 94\%, while inter-examiner agreement was excellent at $\kappa=0.915$ (95\% CI 0.688-1.00). At baseline $(n=35)$, the mean severity of AC lesions was $3.06( \pm$ $0.99)$, with $45.7 \%$ of participants $(n=16)$ having grade IV and $34,3 \%(n=12)$ grades I or II. Over the observation period, mean severity was $2.4( \pm 1.10)$ at 30 days $(\mathrm{n}=$ $32), 2.2( \pm 1.24)$ at 60 days $(\mathrm{n}=32)$, and $2.0( \pm 1.51)$ at 90 days $(n=29)$ (Table 2$)$. A progressive reduction in mean AC severity was thus observed, especially between baseline and 90 days (from 3.06 to 2.03 respectively).

Table 1: Distribution of sociodemographic, behavioral, and clinical variables of study participants $(\mathrm{n}=35)$.

\begin{tabular}{|c|c|c|}
\hline Variable & Category & n (\%) \\
\hline \multirow[t]{2}{*}{ Sex } & Male & $16(45.7 \%)$ \\
\hline & Female & $19(54.3 \%)$ \\
\hline \multirow[t]{2}{*}{ Age (years) } & Mean \pm standard deviation & $56.6 \pm 13.66$ \\
\hline & Range & $31-77$ \\
\hline \multirow[t]{2}{*}{ Skin color } & White & $32(91.4)$ \\
\hline & Brown & $3(8.6)$ \\
\hline \multirow[t]{3}{*}{ Educational attainment } & Primary or less & $14(40)$ \\
\hline & Secondary or less & $17(48.6)$ \\
\hline & Higher & $4(11.5)$ \\
\hline \multirow[t]{3}{*}{ Monthly income } & $\leq 1 \times$ minimum wage & $11(31.4)$ \\
\hline & $1-3 \times$ minimum wage & $13(37.1)$ \\
\hline & $>3 \times$ minimum wage & $11(31.5)$ \\
\hline \multirow[t]{3}{*}{ Duration of sun exposure (years) } & $\leq 8$ & $3(12.9)$ \\
\hline & $10-20$ & $8(34.8)$ \\
\hline & $>20$ & $12(52.2)$ \\
\hline \multirow[t]{3}{*}{ Smoking } & Current & $19(54.3)$ \\
\hline & Never & $3(8.6)$ \\
\hline & Former & $13(37.1)$ \\
\hline \multirow[t]{2}{*}{ Comorbidities } & Yes & $23(65.7)$ \\
\hline & No & $12(34.3)$ \\
\hline \multirow[t]{3}{*}{ Alcohol intake } & Current & $3(8.6)$ \\
\hline & Never & $28(80.0)$ \\
\hline & Former & $4(11.4)$ \\
\hline
\end{tabular}

Table 2: Distribution of severity of actinic cheilitis lesions at baseline and throughout the study period.

\begin{tabular}{|l|c|c|c|c|c|}
\hline Time point & $n$ & $\begin{array}{c}\text { Grade, } \\
\text { Mean }\end{array}$ & $\begin{array}{c}\text { Grade, } \\
\text { Median }\end{array}$ & SD & $\begin{array}{c}\text { Grade, } \\
\text { Min-Max }\end{array}$ \\
\hline Baseline & 35 & 3.06 & 3.0 & 0.99 & $1-4$ \\
\hline 30-day follow-up & 32 & 2.47 & 2.0 & 1.01 & $0-4$ \\
\hline 60-day follow-up & 32 & 2.25 & 2.0 & 1.24 & $0-4$ \\
\hline 90-day follow-up & 29 & 2.03 & 3.0 & 1.51 & $0-4$ \\
\hline
\end{tabular}

SD: standard deviation. 
Comparison of mean severity at baseline and at 30, 60, and 90 days showed a significant difference in terms of disease regression ( $p=0.000)$, starting at 30 days of treatment (Table 3). The largest difference was found on comparison of baseline versus the 90-day end-of-study visit $(p=0.000)$. to cumulative UVR exposure. This effect is attributable to a reduction in the stratum corneum and restoration of the dermis within 120 days of treatment $(12,13)$. This may perhaps explain why the 90-day observation period used in the present study was insufficient; use of the barrier cream for a longer period might have allowed the

Table 3: Distribution of mean values and mean differences in actinic cheilitis severity at baseline and follow-up of participants who completed the study period $(n=29)$.

\begin{tabular}{|c|c|c|c|c|c|}
\hline \multicolumn{3}{|c|}{ Time point (Mean grade) } & \multirow{2}{*}{$\begin{array}{c}\text { Difference in means } \\
0.66 \mathrm{a} \\
\end{array}$} & \multirow{2}{*}{$\begin{array}{c}\text { Bonferroni Sig.* } \\
0.000\end{array}$} & \multirow{2}{*}{$\begin{array}{c}\mathbf{9 5} \% \mathrm{CI} \\
0.22-1.09 \\
\end{array}$} \\
\hline Baseline (3.0) & & 30 days (2.34) & & & \\
\hline & $\begin{array}{l}3 \\
3 \\
3\end{array}$ & 60 days $(2.17)$ & $0.83 \mathrm{a}$ & 0.000 & $0.31-1.34$ \\
\hline & $\begin{array}{l}\stackrel{0}{\bar{O}} \\
\text { 王 }\end{array}$ & 90 days $(2.0)$ & $1.0 \mathrm{a}$ & 0.000 & $0.38-1.62$ \\
\hline
\end{tabular}

Of the participants who completed the study $(\mathrm{n}=29)$, $27.6 \%(n=8)$ had experienced no change in AC severity from baseline at 90 days. Three participants $(10.3 \%)$ had worsening $\mathrm{AC}$ and were referred for biopsy. More than half of the sample $(62 \%)$ had complete $(n=9)$ or partial $(\mathrm{n}=9)$ remission of $\mathrm{AC}$ lesions.

There was a correlation between improvement in $\mathrm{AC}$ severity and participant gender, with worse responses being observed in males $(p=0.002)$. Furthermore, the longer the duration of sun exposure in years, the lower the rate of remission ( $p=0.007)$. Nevertheless, no correlation was found with participant age $(p=0.138)$ nor with behavioral habits, including smoking $(p=0.930)$.

\section{Discussion}

The results of this study showed that daily use of a barrier cream containing vitamins $\mathrm{A}, \mathrm{D}, \mathrm{E}$ and $\mathrm{ZnO}$ had a progressive effect leading to complete or partial remission of lower-lip AC lesions in more than half of the sample $(62 \%)$, regardless of baseline severity. The 3 -month period of observation was not sufficient for complete remission of AC; however, it bears stressing that about $46 \%$ of participants had grade IV lesions at baseline. On the other hand, approximately $34 \%$ of participants with grade IV lesions at baseline experienced two to four grades of improvement in clinical severity at the end of the 90-day period. In addition, baseline participants with severity grades I-III accounted for the highest proportion of cases achieving complete remission at 90 days.

This effect on the remission of AC lesions may be attributable to the components of the barrier cream, especially retinoic acid (vitamin $\mathrm{A}$ ), which has been shown to have a positive effect on photodamage in UVR-exposed skin (12). Tretinoin, another vitamin A derivative, has been shown to reverse the effects of photoaging, with a $23-60 \%$ progressive reduction in actinic keratoses due full effect of the retinol component to manifest itself, with the possibility of complete remission of AC lesions. Vitamin D, another component of the formula, in addition to keeping the lips hydrated and protected, may have acted synergistically with vitamin A. When combined with 5-fluorouracil (5-FU) in the treatment of actinic keratoses, this vitamin has been shown to reduce the number of lesions and minimize adverse events of 5-FU, such as pain, scabbing, and skin ulceration, which also improves treatment tolerability (17).

In the present study, cases classified as grade IV with areas of erosion had a rapid response to treatment. This effect is probably attributable to $\mathrm{ZnO}$, another component of the formulation. Zinc compounds are known to increase the potential for keratinocyte migration and collagenolytic activity, in addition to stimulating tissue reepithelization and repair (20). In addition, $\mathrm{ZnO}$-containing creams have been shown to induce complete remission of dermatitis very rapidly (after approximately 4 days of use) (22).

A systematic review (11) compared different treatments for AC lesions and concluded that the combination of imiquimod with photodynamic therapy (PDT) improved clinical appearance in 80 to $100 \%$ of cases. However, it is noteworthy that most of the included studies had no formal sample size calculation and enrolled a small number of participants, which may have led to random error and directly impacted the methodological quality and heterogeneity of the available literature regarding the effectiveness of treatments for AC. It should also be noted that, in this review, the use of PDT with imiquimod was implicated in a wide range of adverse effects, including itching, pain, redness, swelling, blistering and crusting, and superficial erosions and ulcerations (8). In the present study, participants only reported superficial lip desquamation in the first days of treatment. 
The results of our study cannot be compared to those of invasive treatments for AC. However, there are several less invasive, easy-to-use, low-cost alternatives that can both prevent and treat AC. This is especially relevant considering as verified in the present study - the potential for progressive remission of severe AC lesions, which pose the highest risk of malignant transformation to SCC (21). In our investigation, we found significant correlations between lower remission rates and longer duration of sun exposure (in years), only among male participants. Possible explanations of this result include the lower adherence of men to treatments that require continuous use (23). Also, one must consider the cumulative effects of UVR exposure to the lip epithelium, which may lead to decreased responsiveness and tissue repair $(1,6,24)$.

Possible limitations of this study include the fact that clinical examination may sometimes underestimate the severity of AC in cases with a less aggressive appearance and varying degrees of undetectable dysplasia. However, this possible confounding factor was minimized by clinical classification of lesion severity, based on well-established criteria (21). Other limitation, only patients of 43 years or older were include in the study. At the end of the 90-day observation period, despite the loss of follow-up of some participants $(n=6)$, a number close to that established by the sample size calculation remained. Thus, our study was able to detect a 0.66 -grade difference in AC severity between baseline and the end of the study. This can be considered a strength of the present study, as can the prospective follow-up of the participants - which allowed detection of clinical changes at any time and, especially, the possibility of comparing cases before and after the start of the treatment. Nevertheless, the promising results obtained in this preliminary study with use of a vitamin-based topical therapy for $\mathrm{AC}$, create a perspective for further research. Future studies should focus on longer treatment and follow-up periods and enroll comparator groups to ascertain the efficacy and effectiveness of this treatment.

\section{Conclusions}

Daily topical use of vitamin-based barrier cream has a promising positive impact on the clinical severity of actinic cheilitis. However, a 90-day course of treatment was not sufficient to achieve complete remission. The findings of this study suggest a promising new avenue for the treatment of lower-lip AC.

\section{References}

1. Rodríguez- Blanco I, Flórez Á, Paredes-Suárez C, Rodríguez-Lojo R, González-Vilas D, Ramírez-Santos A, et al. Actinic Cheilitis Prevalence and Risk Factors: A Cross-sectional, Multicentre Study in a Population Aged 45 Years and Over in North-west Spain. Acta Derm Venereol. 2018;98:970-974.

2. Dancyger A, Heard V, Huang B, Suley C, Tang D, Ariyawardana A. Malignant transformation of actinic cheilitis: A systematic review of observational studies. J Invest Clin Dent. 2018;4:e12343.
3. Lupu M, Caruntu A, Caruntu C, Boda D, Moraru L, Voiculescu V, et al. Non-invasive imaging of actinic cheilitis and squamous cell carcinoma of the lip. Mol Clin Oncol. 2018;8:640-646.

4. van der Waal I. Potentially malignant disorders of the oral and oropharyngeal mucosa; terminology, classification and present concepts of management. Oral Oncol. 2009;45:317-23.

5. de Oliveira Ribeiro A, da Silva LC, Martins-Filho PR. Prevalence of and risk factors for actinic cheilitis in Brazilian fishermen and women. Int J Dermatol. 2014;53:1370-1376.

6. Gheno JN, Martins MA, Munerato MC, Hugo FN, Sant'ana Filho $\mathrm{M}$, Weissheimer $\mathrm{C}$, et al. Oral mucosal lesions and their association with sociodemographic, behavioral, and health status factors. Braz Oral Res. 2015;29:1-6.

7. Rodríguez- Blanco I, Flórez Á, Paredes-Suárez C, Rodríguez-Lojo R, González-Vilas D, Ramírez-Santos A, et al. Use of lip photoprotection in patients suffering from actinic cheilitis. Eur J Dermatol. 2019;29:383-386

8. Sotiriou E, Lallas A, Goussi C, Apalla Z, Trigoni A, Chovarda E, et al. Sequential use of photodynamic therapy and imiquimod $5 \%$ cream for the treatment of actinic cheilitis: a 12-month follow-up study. $\mathrm{Br} \mathrm{J}$ Dermatol. 2011;165:888-892.

9. Gupta AK, Paquet M, Villanueva E, Brintnell W. Interventions for actinic keratoses Cochrane Database Syst Rev. 2012;12:CD004415.

10. Choi SH, Kim KH, Song KH. Efficacy of ablative fractional laser-assisted photodynamic therapy for the treatment of actinic cheilitis: 12-month follow-up results of a prospective, randomized, comparative trial. Br J Dermatol. 2015;173:184-191.

11. Salgueiro AP, Jesus LH, Souza IF, Rados PV, Visioli F. Treatment of actinic cheilitis: a systematic review. Clin Oral Invest. 2019;23:20412053.

12. Ianhez M, Pinto SA, Miot HA, Bagatin E. A randomized, open, controlled trial of tretinoin $0.05 \%$ cream vs. low-dose oral isotretinoin for the treatment of field cancerization. Int J Dermatol. 2019;58:365373.

13. Sumita JM, Miot HA, Soares JLM, Raminelli ACP, Pereira SM, Ogawa MM, et al. Tretinoin ( $0.05 \%$ cream vs. $5 \%$ peel) for photoaging and field cancerization of the forearms: randomized, evaluator-blinded, clinical trial. J Eur Acad Dermatol Venereol. 2018;32:1819-1826. 14. McDaniel DH, Mazur C, Wortzman MS, Nelson DB. Efficacy and tolerability of a double-conjugated retinoid cream vs $1.0 \%$ retinol cream or $0.025 \%$ tretinoin cream in subjects with mild to severe photoaging. J Cosmet Dermatol. 2017;16:542-548.

15. Langberg M, Rotem C, Fenig E, Koren R, Ravid A. Vitamin D protects keratinocytes from deleterious effects of ionizing radiation. Br J Dermatol. 2009;160:151-161.

16. Russell M. Assessing the relationship between vitamin D3 and stratum corneum hydration for the treatment of xerotic skin. Nutrients. 2012;4:1213-1218.

17. Cunningham TJ, Tabacchi M, Eliane JP, Tuchayi SM, Manivasagam S, Mirzaalian H, et al. Randomized trial of calcipotriol combined with 5-fluorouracil for skin cancer precursor immunotherapy. J Clin Invest. 2017;127:106-116.

18. Grimm M, Cetindis M, Biegner T, Lehman M, Munz A, Teriete P, et al. Serum vitamin D levels of patients with oral squamous cell carcino$\mathrm{ma}$ (OSCC) and expression of vitamin $\mathrm{D}$ receptor in oral precancerous lesions and OSCC. Med Oral Patol Oral Cir Bucal. 2015;20:e188-95. 19. Pedrelli VF, Lauriola MM, Pigatto PD. Clinical evaluation of photoprotective effect by a topical antioxidants combination (tocopherols and tocotrienols). J Eur Acad Dermatol Venereol. 2012;26:14491453 .

20. Lansdown AB, Mirastschijski U, Stubbs N, Scanlon E, Agren MS. Zinc in wound healing: Theoretical, experimental, and clinical aspects. Wound Rep Reg. 2007;15:2-16.

21. Poitevin NA, Rodrigues MS, Weigert KL, Macedo CLR, Dos Santos RB. Actinic cheilitis: proposition and reproducibility of a clinical criterion. BDJ Open. 2017;3:17016.

22. Chaithirayanon S. Comparative Study between Talcum and Zinc Oxide Cream for the Prevention of Irritant Contact Diaper Dermatitis in Infants. J Med Assoc Thai. 2016; 99, Suppl. 8:S1-S6. 
23. Dalvi V, Mekoth N. Regimen Difficulty and Medication Non-Adherence and the Interaction Effects of Gender and Age. Hosp Top. 2018;96:235-241.

24. Ferreira AM, de Souza Lucena EE, de Oliveira TC, da Silveira É, de Oliveira PT, de Lima KC. Prevalence and factors associated with oral potentially malignant disorders in Brazil's rural workers. Oral Dis. 2016;22:536-542.

\section{Ethies}

All procedures performed in studies involving human participants were in accordance with the national research ethics committee of Brazil, CAAE: 89399618.9.0000.53490, protocol n0 2,751,893) and with the 1964 Helsinki declaration and its later amendments.

\section{Funding}

All costs of this research were borne by the investigators, with no ties to or funding from manufacturers or suppliers.

\section{Authors' Contributions}

All authors contributed to the study conception and design. Formal analysis and investigation: Mariana Sudati Rodrigues, Juliana Tomaz Sganzerla, Kelda Zanchi Younan; Eduardo Oliveira Kraefer; Analysis of results: Ruben Beraldo dos Santos, Pedro Antonio González Hernández, Writing - original draft preparation: Mariana Sudati Rodrigues, Humberto Thomazi Gassen; Complete manuscript review: Sergio Augusto Quevedo Miguens-Jr.

\section{Conflict of interest}

The authors declare that they have no conflict of interest. 